\title{
Quadratic Variational Framework for Signal Design on the 2-Sphere
}

\author{
Liying Wei, Member, IEEE, Rodney A. Kennedy, Fellow, IEEE, and Tharaka A. Lamahewa, Member, IEEE
}

\begin{abstract}
This paper introduces a quadratic variational framework for solving a broad class of signal design problems on the 2-sphere. The functional, to be extremized, combines energy concentration measures using a weighting function in the spatial domain, multiplicative weights in the spectral domain, and a total energy constraint. This leads to two formulations of the signal design problem on the 2 -sphere, one a Fredholm integral equation in the spatial domain and the other an infinite matrix equation in the spectral domain. The framework is illustrated by deriving the key equations for the two classical spatio-spectral concentration problems on the 2-sphere, and for an isotropic filter design that maximizes the filtered energy. In addition, using the proposed framework, we formulate a joint 3-D beamforming application which achieves optimal directivity and spatial resolution simultaneously.
\end{abstract}

Index Terms-Directional derivative, isotropic convolution, uncertainty principle, unit sphere.

\section{INTRODUCTION}

A CCORDING to Fourier transform, a signal cannot sharply confine itself both in the time (spatial) domain and the frequency (spectral) domain. Fraction-out-of-band (FOBE) is a well known measure to the degree of concentration of energy simultaneously in the time-frequency domain [1]-[3]. Paper [1] only considered the finite time interval concentration problem for a band-limited signal and derived the optimal functions - prolate spheroidal wave functions (PSWFs). Meanwhile [2] solved a more general concentration problem for an arbitrary signal which is neither band-limited nor time-limited. Later, a quadratic variational framework by jointly time-frequency concentration measure was developed by Franks in [4]. This framework not only generalizes the work by Slepian-Landau-Pollak [1], [2], but also subsumes many other optimization problems expressed in terms of weighted energy functionals, such as optimal waveform design to achieve the minimum error rate [5], [6], efficient bandwidth usage [7], [8] and high-resolution for signal detection [9], [10].

Manuscript received October 01, 2010; revised February 08, 2011 and May 17, 2011; accepted July 06, 2011. Date of publication July 22, 2011; date of current version October 12, 2011. The associate editor coordinating the review of this manuscript and approving it for publication was Dr. Soontorn Oraintara. This work was supported by the Australian Research Council Discovery Grant DP1094350.

L. Wei was with the Research School of Engineering, College of Engineering and Computer Science, The Australian National University, Canberra, ACT 0200, Australia. She is currently with the Department of Electronic Engineering, The Chinese University of Hong Kong, Hong Kong (e-mail: lywei@ee.cuhk. edu.hk).

R. A. Kennedy and T. A. Lamahewa are with the Research School of Engineering, College of Engineering and Computer Science, The Australian National University, Canberra, ACT 0200, Australia (e-mail: rodney.kennedy@anu.edu.au; tharaka.lamahewa@anu.edu.au).

Digital Object Identifier 10.1109/TSP.2011.2162506
Compared with signal design in time-frequency domain, signal processing on the unit sphere, $\mathbb{S}^{2}$, also called the 2-sphere, which relates to the spatial-spectral domain, is a relatively under-explored area. Though recently, more and more applications on the 2-sphere are developing, such as optimal filter design for filtering and surface smoothing in computer vision [11], [12], detection of compact objects embedded in the stochastic background process [13]-[15], power spectrum estimation in cosmology [16]-[19] and wireless channel modeling and 3-D beamforming/sensing [20]-[22]. However, these works only consider band-limited or spectral-limited signals.

Analogous to Slepian-Landau-Pollak concentration problem in time-frequency domain, [23]-[34] have developed some results on the 2 -sphere by considering different energy concentration measures in the spatial-spectral domain. However, most of these published work only consider the energy concentration measure for special signals under different concentration criteria, i.e., the spatial concentration of a spectral-limited signal [25], [27], [28], [32], [33] and the spectral concentration of a spatially limited signal [26], [28], [34]. These works adopted special weighting functions in the spatial domain (e.g., a box-car window function) [25], [27], [28], [31] or a weighting sequences in the spectral domain (e.g., a set of finite identity sequence with each element "1") [26], [28]. Few works have been published relating to arbitrary weighting functions or weighting sequences [12], [32]-[36]. In these works, we note that the choices of the concentration criteria are determined either by the weighting functions in the spatial domain or the weighting sequences in the spectral domain. Note that aforementioned works only consider either the case of a spatial limited signal or a spectral limited signal. Therefore, a discussion related to an arbitrary spherical signal's simultaneously concentration measure in the spatio-spectral domain is necessary.

As we pointed out before, Franks general variational framework formulated an optimization problem by minimizing the sum of time and frequency concentration measures, which not only generalized the truly jointly time-frequency concentration problem of Slepian-Landau-Pollak [1], [2], [4], but also unified other optimization problems relating to arbitrarily weighted time-frequency energy concentration measure. However, no common unifying framework relating to spatial-spectral domain using arbitrarily weighting functions both in the spatial domain and in the spectral domain, analogous to the Franks time-frequency quadratic framework, has been developed. Therefore, in this paper, we aim to develop an analogous quadratic variational framework on the 2-sphere which can unify many relevant signal design problem expressible by a pair of weighting functions in the spatial and spectral domains, including truly jointly spatio-spectral concentration problem. The main contributions of the paper are summarized as follows. 
- We develop a quadratic variation framework by extremizing the sum of arbitrarily weighted spatial and spectral energy concentration measures, which can unify most of optimization problems relating to different energy concentration criteria on spatial-spectral domain. More importantly, this framework not only generalizes the special work in [28], but also provides a framework to solve the jointly spatio-spectral optimization problem for an arbitrary signal.

- We derive the relationship between a kernel function and its corresponding infinite matrix representation for a bounded linear operator. This sets up the mechanism to move back and forth between the spatial and spectral domains for an operator operation.

- We demonstrate the strength of the proposed framework by applying it to two practical applications found in computer vision, geophysics and wireless communications: in Section IV-C we formulate an isotropic filter with Gauss-Weierstrass kernel and find an orthogonal set of optimally input functions that maximize the output energy, and in Section IV-D we formulate a joint 3-D beamforming design, which achieves optimal directivity and spatial resolution simultaneously.

The rest of the paper is organized as follows. Section II provides notation and some mathematical preliminaries for signals defined on the 2-sphere. Section III formulates our quadratic variational framework with three quadratic functionals relating to energy concentration by a pair of spatial and spectral weighting functions. In this section, a spherical harmonic multiplication operator which includes the isotropic convolution as a special case is implemented as the spectral weighting. This section also deduces the infinite matrix representations and the kernel functions of some special bounded linear operators that characterize the objective functionals in the quadratic variational framework. Using the directional derivative of the objective function, the necessary conditions of a stationary point to the quadratic variational problem in both spatial domain and spectral domain are derived. In Section IV, we demonstrate the applications of our quadratic framework by applying it to spatio-spectral concentration work presented in [28]. Furthermore, this section provides two examples using our framework: 1) obtain the optimal input signal achieving the maximum filtered energy for a parameterized linear system, and 2) design a joint 3-D beamforming scheme. Finally, the conclusions are given in Section V.

\section{Mathematical Preliminaries}

\section{A. Notations}

Let $\mathbb{S}^{2}=\left\{\boldsymbol{x} \in \mathbb{R}^{3}:\|\boldsymbol{x}\|=1\right\}$ denote the unit sphere in $\mathbb{R}^{3}$, where $\boldsymbol{x} \equiv(\theta, \phi) \triangleq(\sin \theta \cos \phi, \sin \theta \sin \phi, \cos \theta)$ denotes a point on the sphere, $\theta \in[0, \pi)$ is the co-latitude and $\phi \in[0,2 \pi]$ is the longitude.

Let $L^{2}\left(\mathbb{S}^{2}\right)$ be a complex Hilbert space containing all the square-integrable functions on the sphere $\mathbb{S}^{2}$ with inner product

$$
\langle f, g\rangle=\int_{\mathbb{S}^{2}} f(\boldsymbol{x}) \overline{g(\boldsymbol{x})} d s(\boldsymbol{x})
$$

$$
=\int_{0}^{\pi} \int_{0}^{2 \pi} f(\theta, \phi) \overline{g(\theta, \phi)} \sin \theta d \phi d \theta
$$

where $d s(\boldsymbol{x})=\sin \theta d \theta d \phi$ and $\overline{[\cdot]}$ denotes complex conjugation. The inner product induces a norm, $\|f\|=\langle f, f\rangle^{1 / 2}$.

\section{B. Spherical Harmonics and Fourier Representation}

The spherical harmonics $Y_{n}^{m}(\boldsymbol{x}) \equiv Y_{n}^{m}(\theta, \phi)$ are defined by [37]

$$
\begin{aligned}
& Y_{n}^{m}(\theta, \phi)=\sqrt{\frac{2 n+1}{4 \pi} \frac{(n-m) !}{(n+m) !}} P_{n}^{m}(\cos \theta) e^{i m \phi}, \\
& n=0,1, \ldots, \quad m=-n, \ldots, n
\end{aligned}
$$

where $P_{n}^{m}(\cdot)$ are the associated Legendre functions, $n$ is the angular (spectral) degree, $m(-n \leq m \leq n)$ is the angular order and $i=\sqrt{-1}$.

As $Y_{n}^{m}(\boldsymbol{x})$ form a complete, orthonormal basis in $L^{2}\left(\mathbb{S}^{2}\right)$, any finite energy signal $f \in L^{2}\left(\mathbb{S}^{2}\right)$ can be represented, in the sense of convergence in the mean with the induced norm, by

$$
f(\boldsymbol{x})=\sum_{n=0}^{\infty} \sum_{m=-n}^{n} f_{n}^{m} Y_{n}^{m}(\boldsymbol{x}),
$$

where the spherical harmonic Fourier coefficients $f_{n}^{m}$ are given by

$$
f_{n}^{m}=\left\langle f, Y_{n}^{m}\right\rangle=\int_{\mathbb{S}^{2}} f(\boldsymbol{x}) \overline{Y_{n}^{m}(\boldsymbol{x})} d s(\boldsymbol{x}) .
$$

\section{PROBlem Formulation}

The quadratic variational problem on the 2 -sphere is to find an optimal function $f \in L^{2}\left(\mathbb{S}^{2}\right)$ that extremizes the quadratic functional

$$
G=\mu_{1} I_{1}+\mu_{2} I_{2}+I_{3}
$$

with

$$
\begin{aligned}
& I_{1} \triangleq\langle w f, f\rangle=\int_{\mathbb{S}^{2}} w(\boldsymbol{x})|f(\boldsymbol{x})|^{2} d s(\boldsymbol{x}) \\
& I_{2} \triangleq\left\langle\mathcal{K}_{v} f, f\right\rangle=\sum_{n=0}^{\infty} \sum_{m=-n}^{n} v_{n}^{m}\left|f_{n}^{m}\right|^{2} \\
& I_{3} \triangleq\langle f, f\rangle=\|f\|^{2}
\end{aligned}
$$

where $w(\boldsymbol{x}) \in L^{2}\left(\mathbb{S}^{2}\right)$ defines a spatial real, positive multiplicative weighting for functional $I_{1}, \mathcal{K}_{v}$ is a spherical harmonic multiplication operator which provides a spectral real non-negative spectral weighting for functional $I_{2}$ with weights $v_{n}^{m} \geq 0$ for all valid $n$ and $m$, the functional $I_{3}$ is the signal energy, and $\mu_{1}$ and $\mu_{2}$ are Lagrange multipliers. Note that $f(\boldsymbol{x})$ can be a spatial-limited, a spectral-limited signal or an arbitrary signal.

The above problem formulation draws direct analogy to the framework developed by Franks to study a broad class of signal concentration and signal design problems in time and frequency [4, ch. 6]. In [4], the analogy of $I_{1}$ measures time domain energy concentration, the analogy of $I_{2}$ measures frequency domain energy concentration and $I_{3}$ is the energy content of a 
signal energy. Analogous to framework of Franks in time-frequency, our primary aim is to introduce a quadratic variational framework for solving a broad class of signal design problems on the 2-sphere by measuring the weighted energy concentration in terms of arbitrary weighting functions both in the spatial domain and the spectral domain, which is represented by the spherical spectral Fourier coefficients.

\section{A. Solutions to Quadratic Variational Framework}

To solve the quadratic variational problem (3), both necessary conditions and sufficient conditions to a stationary point are required. Usually, the sufficiency conditions of a particular stationary point relate to the specified physical considerations that led to the formulation of the problem [4]. Therefore, after solving the necessary conditions, one still needs to determine the Lagrange multipliers $\mu_{1}$ and $\mu_{2}$ that simultaneously extremize the performance functional and satisfy the constraint equations, which is another extremization problem and often requires numerical methods to obtain the final solution. In this paper, we focus on the necessary conditions of a stationary point to the quadratic variational problem.

\section{B. Spherical Harmonic Multiplication Operator}

In this section, we provide detailed information of the operator $\mathcal{K}_{v}$ given in (4b). It is developed as a convenient mathematical tool to weight the functions of interest in the spectral domain, but needs careful interpretation. Putting aside the physical requirement (non-negative weighting, or $v_{n}^{m} \geq 0$ ), this operator is very convenient for signal analysis in practical applications, especially as it subsumes the special class of isotropic convolution operators.

The harmonic multiplication operator is defined by [38]

$$
\left(\mathcal{K}_{v} f\right)(\boldsymbol{x})=\int_{\mathbb{S}^{2}} K_{v}(\boldsymbol{x}, \boldsymbol{y}) f(\boldsymbol{y}) d s(\boldsymbol{y}), \quad \boldsymbol{x} \in \mathbb{S}^{2}
$$

where the kernel function $K_{v}(\boldsymbol{x}, \boldsymbol{y})$ is given by

$$
K_{v}(\boldsymbol{x}, \boldsymbol{y})=\sum_{n=0}^{\infty} \sum_{m=-n}^{n} v_{n}^{m} Y_{n}^{m}(\boldsymbol{x}) \overline{Y_{n}^{m}(\boldsymbol{y})} .
$$

As $v_{n}^{m}$ are real, so we have $K_{v}(\boldsymbol{x}, \boldsymbol{y})=\overline{K_{v}(\boldsymbol{y}, \boldsymbol{x})}$. Therefore, $\mathcal{K}_{v}$ is a self-adjoint operator. In addition, we have $v_{n}^{m}$ non-negative, with at least one positive.

After substituting (6) into (5), and combining (2), we have [38]

$$
\left(\mathcal{K}_{v} f\right)(\boldsymbol{x})=\sum_{n=0}^{\infty} \sum_{m=-n}^{n} v_{n}^{m} f_{n}^{m} Y_{n}^{m}(\boldsymbol{x}), \quad \boldsymbol{x} \in \mathbb{S}^{2} .
$$

1) Special Case-Equivalence to Isotropic Convolution: Taking $v_{n}^{m}=v_{n}$ for all $n$ and $m$, that is, $v_{n}$ is a function only of $n$, we have

$$
\left(\mathcal{K}_{v} f\right)(\boldsymbol{x})=\sum_{n=0}^{\infty} \sum_{m=-n}^{n} v_{n} f_{n}^{m} Y_{n}^{m}(\boldsymbol{x}), \quad \boldsymbol{x} \in \mathbb{S}^{2},
$$

and the kernel can be expressed by

$$
K_{v}(\boldsymbol{x}, \boldsymbol{y})=\sum_{n=0}^{\infty} v_{n} \frac{2 n+1}{4 \pi} P_{n}(\boldsymbol{x} \cdot \boldsymbol{y})
$$

where $\boldsymbol{x} \cdot \boldsymbol{y}$ is the dot product between the unit vectors $\boldsymbol{x}$ and $\boldsymbol{y}$, $P_{n}(\cdot)$ are the Legendre polynomials and the spherical harmonic addition theorem

$$
\sum_{m=-n}^{n} Y_{n}^{m}(\boldsymbol{x}) \overline{Y_{n}^{m}(\boldsymbol{y})}=\frac{2 n+1}{4 \pi} P_{n}(\boldsymbol{x} \cdot \boldsymbol{y})
$$

is used. Looking at (7), $K_{v}(\boldsymbol{x}, \boldsymbol{y})$ only depends on the angle between $\boldsymbol{x}$ and $\boldsymbol{y}$, whence $K_{v}(\boldsymbol{x}, \boldsymbol{y})$ can be also represented as $K_{v}(\boldsymbol{x} \cdot \boldsymbol{y})$.

The isotropic convolution of $f \in L^{2}\left(\mathbb{S}^{2}\right)$ with an axisymmetric function $z(\boldsymbol{x}) \in L^{2}\left(\mathbb{S}^{2}\right)$, using the binary operand symbol $\circledast$, is defined by [16], [38], [39]

$$
\left(\mathcal{I}_{z} f\right)(\boldsymbol{x})=(z \circledast f)(\boldsymbol{x})=\int_{\mathbb{S}^{2}} I_{z}(\boldsymbol{x} \cdot \boldsymbol{y}) f(\boldsymbol{y}) d s(\boldsymbol{y})
$$

where $\mathcal{I}_{z}$ is the isotropic convolution operator and its corresponding kernel function $I_{z}(\boldsymbol{x} \cdot \boldsymbol{y})$ is given by

$$
\begin{aligned}
I_{z}(\boldsymbol{x} \cdot \boldsymbol{y}) & =\sum_{n=0}^{\infty} z_{n}^{0} \sqrt{\frac{4 \pi}{2 n+1}} \sum_{m=-n}^{n} Y_{n}^{m}(\boldsymbol{x}) \overline{Y_{n}^{m}(\boldsymbol{y})} \\
& =\sum_{n=0}^{\infty} z_{n}^{0} \sqrt{\frac{2 n+1}{4 \pi}} P_{n}(\boldsymbol{x} \cdot \boldsymbol{y})
\end{aligned}
$$

where $z_{n}^{0}=\left\langle z, Y_{n}^{0}\right\rangle$. By substituting (9) in (8), we obtain

$$
\left(\mathcal{I}_{z} f\right)(\boldsymbol{x})=\sum_{n=0}^{\infty} \sum_{m=-n}^{n} \sqrt{\frac{4 \pi}{2 n+1}} z_{n}^{0} f_{n}^{m} Y_{n}^{m}(\boldsymbol{x}) .
$$

Comparing the kernel function (7) of the special spherical harmonic multiplication operator with that of the isotropic convolution (9), if we let

$$
v_{n}=z_{n}^{0} \sqrt{\frac{4 \pi}{2 n+1}},
$$

we have $\mathcal{K}_{v} f=\mathcal{I}_{z} f$. That is, the spherical harmonic multiplication in this special case is equivalent to the isotropic convolution, or we can say that the weighting sequence $v_{n}$ can be constructed from an axisymmetric function defined on the 2 -sphere.

\section{Matrix Representation of Bounded Linear Operators}

Theorem 4.2.2 in [40] states that a bounded operator on a separable infinite dimensional Hilbert space can be represented by an infinite matrix. Hence, any bounded linear operator $\mathcal{B}$ defined on $L^{2}\left(\mathbb{S}^{2}\right)$ admits an infinite matrix representation with respect to the spherical harmonics $Y_{n}^{m}(\boldsymbol{x})$ [38],

$$
\mathbf{B}_{n q}^{m p} \triangleq\left\langle\mathcal{B} Y_{q}^{p}, Y_{n}^{m}\right\rangle
$$

for all $n=0,1,2, \ldots, m=-n, \ldots, n$ and $q=0,1,2, \ldots$, $p=-q, \ldots, q$. In (11), we should think of the pair $p, q$ as a single input/column index, and pair $m, n$ as a single output/row index. Note that the coefficient $\mathbf{B}_{n q}^{m p}$ represents how much of $Y_{q}^{p}$ 
as an input gets projected along the $Y_{n}^{m}$ direction of the output under operator $\mathcal{B}$.

In this paper, to obtain the necessary conditions of a stationary point to the objective function (3) in both the spatial domain and spectral domain, we first characterize how a bounded linear operator maps input to output, with respect to the spherical harmonics $Y_{n}^{m}(\boldsymbol{x})$, and then find the matrix representations (spectral domain) and kernel functions (spatial domain) of these operators.

Assume a bounded linear operator $\mathcal{B}$ acts on a function $f \in$ $L^{2}\left(\mathbb{S}^{2}\right)$, i.e., $d=\mathcal{B} f$. Then according to spherical harmonic Fourier transform (2), we have

$$
d_{n}^{m}=\left\langle d, Y_{n}^{m}\right\rangle=\left\langle\mathcal{B} f, Y_{n}^{m}\right\rangle=\sum_{q=0}^{\infty} \sum_{p=-q}^{q} \mathbf{B}_{n q}^{m p} f_{q}^{p} .
$$

Therefore, in spatial form,

$$
(\mathcal{B} f)(\boldsymbol{x})=\sum_{n=0}^{\infty} \sum_{m=-n}^{n} \sum_{q=0}^{\infty} \sum_{p=-q}^{q} \mathbf{B}_{n q}^{m p} f_{q}^{p} Y_{n}^{m}(\boldsymbol{x}) .
$$

1) Relationship Between Kernel Function and Infinite Matrix Representation: To completely characterize a bounded linear operator, it would be convenient to find the relationship between its kernel function and the corresponding infinite matrix representation.

Substituting the kernel function $B(\boldsymbol{x}, \boldsymbol{y})$ in (11), we have

$$
\mathbf{B}_{n q}^{m p}=\int_{\mathbb{S}^{2}}\left(\int_{\mathbb{S}^{2}} B(\boldsymbol{x}, \boldsymbol{y}) Y_{q}^{p}(\boldsymbol{y}) d s(\boldsymbol{y})\right) \overline{Y_{n}^{m}(\boldsymbol{x})} d s(\boldsymbol{x}) .
$$

Using the inverse Fourier transform on the unit sphere (1) and by taking the conjugate on both sides, it can be shown that

$$
B(\boldsymbol{x}, \boldsymbol{y})=\sum_{q=0}^{\infty} \sum_{p=-q}^{q} \sum_{n=0}^{\infty} \sum_{m=-n}^{n} \mathbf{B}_{n q}^{m p} Y_{n}^{m}(\boldsymbol{x}) \overline{Y_{q}^{p}(\boldsymbol{y})}
$$

Remark 1: With (12) and (13), we have the mechanism for moving back and forth between the spatial domain and the spectral domain in characterizing the operator.

We now find the matrix representations and the corresponding kernel functions of the operators present in the quadratic functionals $\left(I_{1}, I_{2}\right.$ and $\left.I_{3}\right)$ composing the objective function (3).

In relation to the spherical harmonic multiplication operator $\mathcal{K}_{v}$, by substituting the kernel function (6) in (12), we have the matrix representation

$$
\mathbf{K}_{n q}^{m p}=v_{q}^{p} \delta_{n}^{q} \delta_{m}^{p}
$$

where $\delta_{n}^{q}\left(\delta_{m}^{p}\right)$ is the Kronecker delta function (equals one if and only if $n=q(m=p)$, and is zero otherwise).

Let $\left(\mathcal{A}_{w} f\right)(\boldsymbol{x})=w(\boldsymbol{x}) f(\boldsymbol{x})$ where $\mathcal{A}_{w}$ is a bounded linear operator. Then according to (11), we have

$$
\begin{aligned}
\mathbf{W}_{n q}^{m p}= & \left\langle\mathcal{A}_{w} Y_{q}^{p}, Y_{n}^{m}\right\rangle \\
= & \sum_{s=0}^{\infty} \sum_{r=-s}^{s} w_{s}^{r}\left[\frac{(2 s+1)(2 q+1)(2 n+1)}{4 \pi}\right]^{1 / 2} \\
& \times\left(\begin{array}{ccc}
n & q & s \\
0 & 0 & 0
\end{array}\right)\left(\begin{array}{ccc}
n & q & s \\
m & p & r
\end{array}\right)
\end{aligned}
$$

where $w_{s}^{r}=\left\langle w, Y_{s}^{r}\right\rangle=\int_{\mathbb{S}^{2}} w(\boldsymbol{x}) \overline{Y_{s}^{r}(\boldsymbol{x})} d s(\boldsymbol{x})$, the arrays of integers are Wigner 3-j symbols [41], and the formula for the integral of a product of three spherical harmonics $Y_{n}^{m}(\boldsymbol{x})$ is used [42],

$$
\begin{aligned}
\int_{\mathbb{S}^{2}} & Y_{s}^{r}(\boldsymbol{x}) Y_{q}^{p}(\boldsymbol{x}) \overline{Y_{n}^{m}(\boldsymbol{x})} d s(\boldsymbol{x}) \\
= & {\left[\frac{(2 s+1)(2 q+1)(2 n+1)}{4 \pi}\right]^{1 / 2} } \\
& \times\left(\begin{array}{ccc}
n & q & s \\
0 & 0 & 0
\end{array}\right)\left(\begin{array}{ccc}
n & q & s \\
m & p & r
\end{array}\right) .
\end{aligned}
$$

Therefore, according to (13), the kernel function of operator $\mathcal{A}_{w}$ is given by

$$
W(\boldsymbol{x}, \boldsymbol{y})=\sum_{q=0}^{\infty} \sum_{p=-q}^{q} \sum_{n=0}^{\infty} \sum_{m=-n}^{n} \mathbf{W}_{n q}^{m p} Y_{n}^{m}(\boldsymbol{x}) \overline{Y_{q}^{p}(\boldsymbol{y})} .
$$

As $w(\boldsymbol{x})$ is real, it can be shown that $W(\boldsymbol{x}, \boldsymbol{y})=\overline{W(\boldsymbol{y}, \boldsymbol{x})}$. As a result, the operator $\mathcal{A}_{w}$ is a self-adjoint operator.

For one special case, $w(\boldsymbol{x})=1$ with $\boldsymbol{x} \in \mathbb{S}^{2}$, then $\mathcal{A}_{w}$ is an identity operator, which we denote by $\mathcal{A}_{\text {Id }}$. The matrix representation in this case be $\mathbf{U}_{n q}^{m p}$ and is given by

$$
\begin{aligned}
\mathbf{U}_{n q}^{m p}= & \delta_{n}^{q} \delta_{m}^{p} \\
= & \sum_{s=0}^{\infty} \sum_{r=-s}^{s} u_{s}^{r}\left[\frac{(2 s+1)(2 q+1)(2 n+1)}{4 \pi}\right]^{1 / 2} \\
& \times\left(\begin{array}{ccc}
n & q & s \\
0 & 0 & 0
\end{array}\right)\left(\begin{array}{ccc}
n & q & s \\
m & p & r
\end{array}\right)
\end{aligned}
$$

where $u_{s}^{r}=\int_{\mathbb{S}^{2}} \overline{Y_{s}^{r}(\boldsymbol{x})} d s(\boldsymbol{x})$. Note that the kernel function $U(\boldsymbol{x}, \boldsymbol{y})$ corresponding to the identity operator $\mathcal{A}_{\mathrm{Id}}$ is the 2-D Dirac delta function $\delta(\boldsymbol{x}, \boldsymbol{y})[42]$, i.e.,

$$
U(\boldsymbol{x}, \boldsymbol{y})=\delta(\boldsymbol{x}, \boldsymbol{y})=\sum_{n=0}^{\infty} \sum_{m=-n}^{n} Y_{n}^{m}(\boldsymbol{x}) \overline{Y_{n}^{m}(\boldsymbol{y})} .
$$

Both expansions (17) and (18) are useful when considering their truncated forms.

Using the matrix representations of operators developed above, the weighting functions in three quadratic functionals $I_{1}, I_{2}$ and $I_{3}$ in (4) can be written as

$$
\begin{aligned}
w(\boldsymbol{x}) f(\boldsymbol{x}) & =\sum_{n=0}^{\infty} \sum_{m=-n}^{n} \sum_{q=0}^{\infty} \sum_{p=-q}^{q} \mathbf{W}_{n q}^{m p} f_{q}^{p} Y_{n}^{m}(\boldsymbol{x}) \\
\left(\mathcal{K}_{v} f\right)(\boldsymbol{x}) & =\sum_{n=0}^{\infty} \sum_{m=-n}^{n} \sum_{q=0}^{\infty} \sum_{p=-q}^{q} \mathbf{K}_{n q}^{m p} f_{q}^{p} Y_{n}^{m}(\boldsymbol{x}) \\
f(\boldsymbol{x}) & =\sum_{n=0}^{\infty} \sum_{m=-n}^{n} \sum_{q=0}^{\infty} \sum_{p=-q}^{q} \mathbf{U}_{n q}^{m p} f_{q}^{p} Y_{n}^{m}(\boldsymbol{x})
\end{aligned}
$$

where $\mathbf{W}_{n q}^{m p}, \mathbf{K}_{n q}^{m p}$, and $\mathbf{U}_{n q}^{m p}$ are the infinite matrix representations of operators $\mathcal{A}_{w}, \mathcal{K}_{v}$, and $\mathcal{A}_{\text {Id }}$, respectively.

\section{Necessary Conditions of Quadratic Variational Framework}

In this section, we aim to find the necessary condition of a stationary solution to the quadratic variational problem (3). Analogous to the time-frequency framework where necessary conditions in both the time domain and frequency domain are pro- 
vided [4], we are interested in finding the necessary condition not only expressed in the spatial domain, but also expressed by the spherical harmonic Fourier coefficients, which can be called the spectral domain for brevity.

In this part, for simplicity, we restrict our attention to real spaces, where all quadratic functionals are real. That is, for a quadratic functional $I(f)=\langle\mathcal{A} f, f\rangle$ with $f \in L^{2}\left(\mathbb{S}^{2}\right)$, where $\mathcal{A}$ is a linear bounded operator with kernel function $A(\boldsymbol{x}, \boldsymbol{y})$, we have $\langle\mathcal{A} f, f\rangle=\langle f, \mathcal{A} f\rangle$. The directional derivative of $I(f)$ at a point $f$ along an arbitrary unit function $u \in L^{2}\left(\mathbb{S}^{2}\right)$ is defined in analogy to [4] as

$$
D_{u} I(f) \triangleq\langle\mathcal{A} u, f\rangle+\langle\mathcal{A} f, u\rangle=\left\langle\left(\mathcal{A}+\mathcal{A}^{\prime}\right) f, u\right\rangle
$$

where $\mathcal{A}^{\prime}$ is the adjoint operator of $\mathcal{A}$ with kernel $A^{\prime}(\boldsymbol{x}, \boldsymbol{y})=$ $\overline{A(\boldsymbol{y}, \boldsymbol{x})} .\left(\mathcal{A}+\mathcal{A}^{\prime}\right) f$ is called the gradient of $I(f)$ at direction $u$, which we denote by $\nabla_{u} I=\left(\mathcal{A}+\mathcal{A}^{\prime}\right) f$. Furthermore, if $\mathcal{A}$ is self-adjoint, i.e., $\mathcal{A}=\mathcal{A}^{\prime}$, then we have $D_{u} I(f)=2\langle\mathcal{A} f, u\rangle$ and $\nabla_{u} I=2 \mathcal{A} f$.

As we have shown that both $\mathcal{K}_{v}$ and $\mathcal{A}_{w}$ are self-adjoint operators, therefore, the directional derivative of (3) can be written as

$$
D_{u} G(f)=2\left\langle\mu_{1} w f+\mu_{2} \mathcal{K}_{v} f+f, u\right\rangle .
$$

A necessary condition for a solution to a quadratic functional $G(f)$ is that the gradient $\nabla_{u} G$ vanishes for all $u \in L^{2}\left(\mathbb{S}^{2}\right)$, i.e., $\nabla_{u} G=0$. Therefore, $\mu_{1} w f+\mu_{2} \mathcal{K}_{v} f+f=0$, or

$\mu_{1} w(\boldsymbol{x}) f(\boldsymbol{x})+\mu_{2} \int_{\mathbb{S}^{2}} K_{v}(\boldsymbol{x}, \boldsymbol{y}) f(\boldsymbol{y}) d s(\boldsymbol{y})+f(\boldsymbol{x})=0, \quad \boldsymbol{x} \in \mathbb{S}^{2}$

where $K_{v}(\boldsymbol{x}, \boldsymbol{y})$ is given by (6). This is the spatial necessary condition for a stationary solution to the quadratic variational problem (3).

Substituting (19a)-(19c) into the spatial necessary condition (20), we obtain

$$
\begin{aligned}
\mu_{1} & \sum_{n=0}^{\infty} \sum_{m=-n}^{n} \sum_{q=0}^{\infty} \sum_{p=-q}^{q} \mathbf{W}_{n q}^{m p} f_{q}^{p} Y_{n}^{m}(\boldsymbol{x}) \\
& +\mu_{2} \sum_{n=0}^{\infty} \sum_{m=-n}^{n} \sum_{q=0}^{\infty} \sum_{p=-q}^{q} \mathbf{K}_{n q}^{m p} f_{q}^{p} Y_{n}^{m}(\boldsymbol{x}) \\
& +\sum_{n=0}^{\infty} \sum_{m=-n}^{n} \sum_{q=0}^{\infty} \sum_{p=-q}^{q} \mathbf{U}_{n q}^{m p} f_{q}^{p} Y_{n}^{m}(\boldsymbol{x})=0
\end{aligned}
$$

Multiplying $\overline{Y_{n}^{m}(\boldsymbol{x})}$ on both sides of the above equation and integrating on the whole sphere $\mathbb{S}^{2}$, we get

$$
\begin{aligned}
\mu_{1} & \sum_{n=0}^{\infty} \sum_{m=-n}^{n} \sum_{q=0}^{\infty} \sum_{p=-q}^{q} \mathbf{W}_{n q}^{m p} f_{q}^{p} \\
& +\mu_{2} \sum_{n=0}^{\infty} \sum_{m=-n}^{n} \sum_{q=0}^{\infty} \sum_{p=-q}^{q} \mathbf{K}_{n q}^{m p} f_{q}^{p} \\
& +\sum_{n=0}^{\infty} \sum_{m=-n}^{n} \sum_{q=0}^{\infty} \sum_{p=-q}^{q} \mathbf{U}_{n q}^{m p} f_{q}^{p}=0
\end{aligned}
$$

where $\mathbf{K}_{n q}^{m p}$ is the infinite matrix representation of the spherical harmonic multiplication operator given by (14), $\mathbf{W}_{n q}^{m p}$ is the infinite matrix representation of the bounded linear operator $\mathcal{A}_{w}$ in (15) and $\mathbf{U}_{n q}^{m p}$, given by (17), is the matrix representation of the operator $\mathcal{A}_{w}$ when $w(\boldsymbol{x})=1$ for all $\boldsymbol{x} \in \mathbb{S}^{2}$. Let $\hat{\mathbf{f}}$ be the column vector containing the spherical harmonic Fourier coefficients $f_{q}^{p}$. Therefore, the spectral necessary condition can be expressed in the matrix form as

$$
\mu_{1} \mathbf{W} \hat{\mathbf{f}}+\mu_{2} \mathbf{K} \hat{\mathbf{f}}+\mathbf{U} \hat{\mathbf{f}}=0
$$

where $\mathbf{W}, \mathbf{K}$, and $\mathbf{U}$ are matrices containing elements $\mathbf{W}_{n q}^{m p}$, $\mathbf{K}_{n q}^{m p}$, and $\mathbf{U}_{n q}^{m p}$, respectively.

\section{APPLICATIONS}

In this section, we demonstrate use of our quadratic variational framework that can unify results for obtaining optimal signals for various energy concentration measures on the 2-sphere.

The classical examples are the spatio-spectral concentration on the 2-sphere [28]. More importantly, applying the equivalence between the spherical harmonic multiplication operation and the isotropic convolution, our framework is a good tool to design optimal waveforms, analogous to time-frequency, to achieve maximum output energy for specified linear filter system, or design matched filter for specified input signal to achieve maximum signal-to-noise ratio, especially for denoising and smoothing signal of interest. In this paper, we provide an example using our framework to obtain the optimal input signal achieving the maximum filtered energy for a linear system [34].

For simplicity, in the following examples, we use the same notation for the operators and infinite matrix representations as the spatial necessary condition equation (20) and the spectral necessary condition equation (21). However, as we have pointed out before, special notes should be given to the integration region and the summation region of spectral degree $n$.

\section{A. Spatial Concentration of a Band-Limited Signal}

The first case is the spatial energy concentration problem for a finite energy spectral-limited signal $f(\boldsymbol{x})=\sum_{n=0}^{N} \sum_{m=-n}^{n} f_{n}^{m} Y_{n}^{m}(\boldsymbol{x})$ in a spatial region $\Gamma \in \mathbb{S}^{2}$. In this case, due to the spectral limitation $n \leq N$, all matrix representations can be taken as finite dimensional, and this is implicit in the notation.

Let

$$
\hat{\mathbf{f}}_{N}=\left(f_{0}^{0}, f_{1}^{0}, \ldots, f_{N}^{N}\right)^{T} .
$$

Using our quadratic variational framework (3), let

$$
v_{n}^{m}= \begin{cases}1, & \forall n \leq N, \quad \forall|m| \leq n \\ 0, & \text { otherwise }\end{cases}
$$

and

$$
w(\mathbf{x})= \begin{cases}1, & x \in \Gamma \in \mathbb{S}^{2}, \\ 0, & \text { otherwise }\end{cases}
$$

Now the problem is to find $f(\boldsymbol{x})$, or equivalently $\hat{\mathbf{f}}$, which maximizes $I_{1}$ subject to constraints $I_{2}$ and $I_{3}$. Note that in this case constraints $I_{2}=I_{3}$ and

$$
\mathbf{K}_{n q}^{m p}=\delta_{n}^{q} \delta_{m}^{p}, \quad \forall n \leq N, \quad \forall|m| \leq n
$$

which is an identity matrix with dimension $(N+1)^{2}$. 
To apply our spectral necessary condition equation (21) in matrix form, as the signal $f(\boldsymbol{x})$ is itself truncated at $N$, let

$$
\begin{gathered}
\hat{\mathbf{f}}=\left(\hat{\mathbf{f}}_{N}, \mathbf{0}\right)^{T}, \quad \mathbf{W}=\left(\begin{array}{ll}
\boldsymbol{W}_{11} & \boldsymbol{W}_{12} \\
\boldsymbol{W}_{21} & \boldsymbol{W}_{22}
\end{array}\right), \\
\mathbf{K}=\left(\begin{array}{ll}
\boldsymbol{K}_{11} & \boldsymbol{K}_{12} \\
\boldsymbol{K}_{21} & \boldsymbol{K}_{22}
\end{array}\right), \quad \mathbf{U}=\left(\begin{array}{ll}
\boldsymbol{U}_{11} & \boldsymbol{U}_{12} \\
\boldsymbol{U}_{21} & \boldsymbol{U}_{22}
\end{array}\right)
\end{gathered}
$$

where $\mathbf{0}$ is a row vector with all elements $0, \boldsymbol{W}_{i j}, \mathbf{K}_{i j}$, and $\boldsymbol{U}_{i j}$ for $1 \leq i, j \leq 2$ are the submatrices containing the elements $\mathbf{W}_{n q}^{m p}, \mathbf{K}_{n q}^{m p}$, and $\mathbf{U}_{n q}^{m p}$, respectively. Especially, the degrees and orders of the elements in $\boldsymbol{W}_{11}, \mathbf{K}_{11}$, and $\boldsymbol{U}_{11}$ are $n \leq N$ and $|m| \leq n$.

Since $\boldsymbol{K}_{12}=\mathbf{0}, \boldsymbol{K}_{21}=\mathbf{0}, \boldsymbol{K}_{22}=\mathbf{0}$, and $\mathbf{U}_{n q}^{m p}=\delta_{n}^{q} \delta_{m}^{p}$, we only need to solve

$$
\mathbf{W}_{11} \hat{\mathbf{f}}_{N}=-\frac{\mu_{2}+1}{\mu_{1}} \hat{\mathbf{f}}_{N}
$$

where $\mathbf{W}_{11}$ is a $(N+1)^{2} \times(N+1)^{2}$ matrix whose elements are given by (15) with $w_{s}^{r}=\left\langle w, Y_{s}^{r}\right\rangle=\int_{\Gamma} \overline{Y_{s}^{r}(\boldsymbol{x})} d s(\boldsymbol{x})$. The detailed derivation of solutions for $f(\boldsymbol{x})$ can be found in [28].

\section{B. Spectral Concentration of a Spatially Limited Signal}

The second case is the spectral energy concentration within bandwidth $N$ for a spatially limited signal $f(\boldsymbol{x})$ with finite energy satisfying $f(\boldsymbol{x})=0$ whenever $\boldsymbol{x} \notin \Gamma \subset \mathbb{S}^{2}$. This variational problem is to find $f(\boldsymbol{x})$ that maximizes $I_{2}$ with $n \leq N$ subject to constraints $I_{1}$ and $I_{3}$. Note that in this case constraints $I_{1}=I_{3}$. We choose the weighting functions: $w(\boldsymbol{x})=1$ with $\boldsymbol{x} \in \Gamma$, and $v_{n}^{m}=1$ for all $0 \leq n \leq N$ and $|m| \leq n$. The bandwidth of $f(\boldsymbol{x})$ is infinite, therefore, only the spatial necessary condition (20) can be applied,

$$
\mu_{1} f(\boldsymbol{x})+\mu_{2} \int_{\Gamma} K_{v}(\boldsymbol{x}, \boldsymbol{y}) f(\boldsymbol{y}) d s(\boldsymbol{y})+f(\boldsymbol{x})=0, \quad \boldsymbol{x} \in \Gamma .
$$

Substituting (6) with $v_{n}^{m}=1$ for any $n \leq N$ and $|m| \leq n$ into above equation, we get

$$
\int_{\Gamma} \sum_{n=0}^{N} \sum_{m=-n}^{n} Y_{n}^{m}(\boldsymbol{x}) \overline{Y_{n}^{m}(\boldsymbol{y})} f(\boldsymbol{y}) d s(\boldsymbol{y})=\left(\frac{-\mu_{1}-1}{\mu_{2}}\right) f(\boldsymbol{x})
$$

which is a Fredholm integral equation of the second kind. The reader is referred to [28] for detailed derivation of solutions for $f(\boldsymbol{x})$.

\section{Maximum Filtered Energy}

In this section, applying the equivalence property of spherical harmonic multiplication operation to the isotropic convolution, we use our framework to find a finite energy, spatially limited function $f(\boldsymbol{x}) \in L^{2}\left(\mathbb{S}^{2}\right)$ for $\boldsymbol{x} \in \Gamma \subset \mathbb{S}^{2}$, which achieves the maximum energy at the output of a specified filter, satisfying (8) with $z(\boldsymbol{x})$ axisymmetric, of bandwidth $N+1$ (i.e., $n \leq N)$, shown in Fig. 1.

With $z(\boldsymbol{x})$ axisymmetric, then

$$
z(\boldsymbol{x})=\sum_{n=0}^{N} z_{n}^{0} Y_{n}^{0}(\boldsymbol{x})
$$

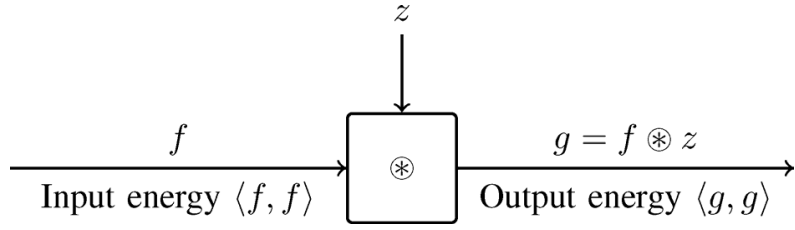

Fig. 1. Isotropic convolution between input signal $f$ and isotropic filter $z$.

where $z_{n}^{0}=\left\langle z, Y_{n}^{0}\right\rangle$. According to the isotropic convolution (10), we have

$$
\begin{aligned}
g(\boldsymbol{x}) & =\left(\mathcal{I}_{z} f\right)(\boldsymbol{x})=z(\boldsymbol{x}) \circledast f(\boldsymbol{x}) \\
& =\int_{\mathbb{S}^{2}} I_{z}(\boldsymbol{x} \cdot \boldsymbol{y}) f(\boldsymbol{y}) d s(\boldsymbol{y}) \\
& =\sum_{n=0}^{N} \sum_{m=-n}^{n} \sqrt{\frac{4 \pi}{2 n+1}} z_{n}^{0} f_{n}^{m} Y_{n}^{m}(\boldsymbol{x})
\end{aligned}
$$

with $I_{z}(\boldsymbol{x} \cdot \boldsymbol{y})=\sum_{n=0}^{\infty} z_{n}^{0} \sqrt{\frac{4 \pi}{2 n+1}} \sum_{m=-n}^{n} Y_{n}^{m}(\boldsymbol{x}) \overline{Y_{n}^{m}(\boldsymbol{y})}$. The output energy is

$$
\|g\|^{2}=\langle g, g\rangle=\left\langle\mathcal{I}_{z} f, \mathcal{I}_{z} f\right\rangle=\left\langle\mathcal{I}_{z}^{\prime} \mathcal{I}_{z} f, f\right\rangle
$$

where $\mathcal{I}_{z}^{\prime}$ is the adjoint operator of $\mathcal{I}_{z}$ with kernel function

$$
\begin{aligned}
I_{z}^{\prime}(\boldsymbol{x}, \boldsymbol{y}) & =\overline{I_{z}(\boldsymbol{y}, \boldsymbol{x})} \\
& =\sum_{n=0}^{\infty} \overline{z_{n}^{0}} \sqrt{\frac{4 \pi}{2 n+1}} \sum_{m=-n}^{n} Y_{n}^{m}(\boldsymbol{x}) \overline{Y_{n}^{m}(\boldsymbol{y})} .
\end{aligned}
$$

Therefore,

$$
\begin{aligned}
\left(\mathcal{I}_{z}^{\prime} \mathcal{I}_{z} f\right)(\boldsymbol{x}) & =\mathcal{I}_{z}^{\prime}\left(\sum_{n=0}^{N} \sum_{m=-n}^{n} \sqrt{\frac{4 \pi}{2 n+1}} z_{n}^{0} f_{n}^{m} Y_{n}^{m}(\boldsymbol{x})\right) \\
& =\sum_{n=0}^{N} \sum_{m=-n}^{n} \sqrt{\frac{4 \pi}{2 n+1}} z_{n}^{0} f_{n}^{m}\left(\mathcal{I}_{z}^{\prime} Y_{n}^{m}\right)(\boldsymbol{x}) \\
& =\sum_{n=0}^{N} \sum_{m=-n}^{n} \frac{4 \pi}{2 n+1}\left|z_{n}^{0}\right|^{2} f_{n}^{m} Y_{n}^{m}(\boldsymbol{x}) .
\end{aligned}
$$

Now the kernel function of $\mathcal{I}_{z}^{\prime} \mathcal{I}_{z}$ is

$$
\begin{aligned}
K_{v}(\boldsymbol{x}, \boldsymbol{y}) & =\sum_{n=0}^{N} \sum_{m=-n}^{n} \frac{4 \pi}{2 n+1}\left|z_{n}^{0}\right|^{2} Y_{n}^{m}(\boldsymbol{x}) \overline{Y_{n}^{m}(\boldsymbol{y})} \\
& =\sum_{n=0}^{N}\left|z_{n}^{0}\right|^{2} P_{n}(\boldsymbol{x} \cdot \boldsymbol{y}) .
\end{aligned}
$$

Obviously, $\mathcal{I}_{z}^{\prime} \mathcal{I}_{z}$ is a self-adjoint operator.

To apply our variational framework (3) to this problem, we let $w(\boldsymbol{x})=1$ with $\boldsymbol{x} \in \Gamma$, then we have $w(\boldsymbol{x}) f(\boldsymbol{x})=f(\boldsymbol{x})$ and $I_{1}=\langle w f, f\rangle$. Further, let $v_{n}^{m}=\frac{4 \pi}{2 n+1}\left|z_{n}^{0}\right|^{2}$ for any $n$ and $m$, and let $\mathcal{K}_{v}=\mathcal{I}_{z}^{\prime} \mathcal{I}_{z}$, where $\mathcal{K}_{v}$ is the spherical harmonic multiplication operator, so we have

$$
I_{2}=\langle g, g\rangle=\left\langle\mathcal{I}_{z}^{\prime} \mathcal{I}_{z} f, f\right\rangle=\left\langle\mathcal{K}_{v} f, f\right\rangle .
$$


Due to finite energy property of $f(\boldsymbol{x})$, let $I_{3}=\langle f, f\rangle=\|f\|^{2}<$ $\infty$. The objective is to maximize the output signal energy $I_{2}$ under the constraints $I_{1}$ and $I_{3}$. Now this problem is completely captured by our framework. Therefore, depending on the explicit filter $z(\boldsymbol{x})$, the spatial necessary condition equation (20) of our framework can be directly used to find the spatially limited function $f(\boldsymbol{x})$ that achieves maximum finite energy at the output of the filter.

As an example, consider the truncated Gauss-Weierstrass kernel [23]

$$
z(\boldsymbol{x})=\sum_{n=0}^{N} \sqrt{\frac{2 n+1}{4 \pi}} e^{-n(n+1) \xi} Y_{n}^{0}(\boldsymbol{x})
$$

where $\xi$ is a constant to control the diffusion variation. Note that the above kernel has been used in [12] for surface smoothing. Comparing (22) with (25) we can clearly see that $z_{n}^{0}=\sqrt{\frac{2 n+1}{4 \pi}} e^{-n(n+1) \xi}$. In the paper [12], though the left convolution [43] is used, the equivalence between the left convolution, denoted by $\star$, and the isotropic convolution (8) has been proved when both filters are the same and axisymmetric, or the kernel functions are univariate [38], i.e.,

$$
\begin{aligned}
\left(\mathcal{I}_{z} f\right)(\boldsymbol{x}) & =\left(\mathcal{R}_{f} z\right)(\boldsymbol{x})=\frac{1}{2 \pi} \int_{\rho \in S O(3)} f(\rho \boldsymbol{\eta}) z\left(\rho^{-1} \boldsymbol{x}\right) d \rho \\
& =\sum_{n=0}^{N} \sum_{m=-n}^{n} \sqrt{\frac{4 \pi}{2 n+1}} z_{n}^{0} f_{n}^{m} Y_{n}^{m}(\boldsymbol{x})
\end{aligned}
$$

where $\mathcal{R}_{f}$ is the left convolution operator, $\rho=(\phi, \theta, \psi)$ is an arbitrary rotation element in $S O(3), \boldsymbol{\eta}=(0,0,1)^{T}$ is the north pole and $d \rho=\sin \theta d \theta d \phi d \psi$ is the Lebesgue measure on $S O(3)$. Comparing (26) with (9) and (6), the kernel function of $\mathcal{R}_{f}$ is

$$
\begin{aligned}
R(\boldsymbol{x}, \boldsymbol{y}) & =\sum_{n=0}^{N} \sum_{m=-n}^{n} z_{n}^{0} \sqrt{\frac{4 \pi}{2 n+1}} Y_{n}^{m}(\boldsymbol{x}) \overline{Y_{n}^{m}(\boldsymbol{y})} \\
& =\sum_{n=0}^{N} \frac{2 n+1}{4 \pi} e^{-n(n+1) \xi} P_{n}(\boldsymbol{x} \cdot \boldsymbol{y})
\end{aligned}
$$

According to (24) and $z_{n}^{0}=\sqrt{\frac{2 n+1}{4 \pi}} e^{-n(n+1) \xi}$, the kernel function of the spherical harmonic multiplication operator $\mathcal{K}_{v}$, or $\mathcal{I}_{z}^{\prime} \mathcal{I}_{z}$ is

$$
K_{v}(\boldsymbol{x}, \boldsymbol{y})=\sum_{n=0}^{N} \sum_{m=-n}^{n} e^{-2 n(n+1) \xi} Y_{n}^{m}(\boldsymbol{x}) \overline{Y_{n}^{m}(\boldsymbol{y})} .
$$

Substituting this into the spatial necessary condition equation (20), we only need to solve

$\int_{\Gamma} \sum_{n=0}^{N} \sum_{m=-n}^{n} e^{-2 n(n+1) \xi} Y_{n}^{m}(\boldsymbol{x}) \overline{Y_{n}^{m}(\boldsymbol{y})} f(\boldsymbol{y}) d s(\boldsymbol{y})=\lambda f(\boldsymbol{x})$

where $\lambda=\frac{-\mu_{1}-1}{\mu_{2}}$. This is equivalent to the variation problem of our former work when $v_{n}^{m}=e^{-n(n+1) \xi}$ for all $n$ and $m$ [34]. Note that $\lambda$ also equals to $\langle g, g\rangle /\langle f, f\rangle$, which is the output energy to input energy ratio. So our problem is to find an optimal function $f(\boldsymbol{x})$ with maximum value of $\lambda$.

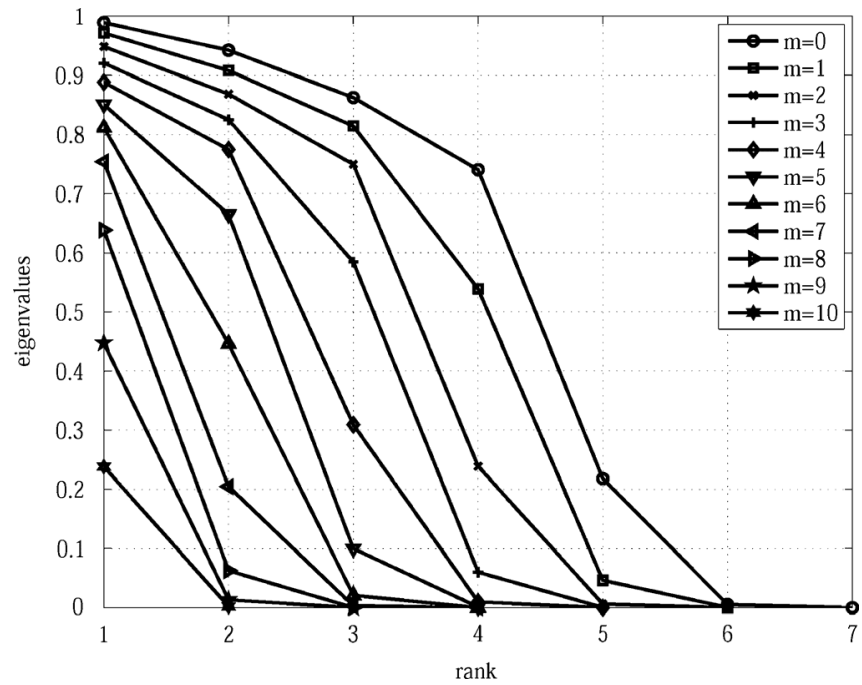

Fig. 2. The distribution of eigenvalues of (28) for $m=0,1, \ldots, 10$ with $x \in \Gamma$, where $\Gamma$ is a polar cap $\left[0,40^{\circ}\right]$, bandwidth $N=18$, and $\xi=5 \times 10^{-4}$. $x$-axis is the rank of eigenvalues for fixed $m, y$-axis is the eigenvalue.

For simplicity, assume the region $\Gamma$ is a polar cap $[0, \Theta]$. Following the same procedure as in our former work [34], let

$$
\begin{aligned}
X_{n}^{m}(\theta) & =\sqrt{\frac{2 n+1}{4 \pi} \frac{(n-m) !}{(n+m) !}} P_{n}^{m}(\cos \theta), \\
f(\theta, \phi) & =\sum_{m=-\infty}^{\infty} e^{i m \phi} \sum_{n=|m|}^{\infty} f_{n}^{m} S_{n}^{m}(\theta)=\sum_{m=-\infty}^{\infty} e^{i m \phi} f_{m}(\theta)
\end{aligned}
$$

where $f_{m}(\theta)=\sum_{n=|m|}^{\infty} f_{n}^{m} S_{n}^{m}(\theta)$ and $Y_{n}^{m}(\theta, \phi)=X_{n}^{m}(\theta) e^{i m \phi}$. After substituting the above equations into (27) and removing the $\phi$ terms on both sides which have no effect in solving the eigenvalue equation problem, we only need to solve a series of fixed-order, one-dimensional homogeneous Fredholm equations of the second kind,

$$
\begin{gathered}
\int_{0}^{\Theta}\left[2 \pi \sum_{n=|m|}^{N} e^{-2 n(n+1) \xi} X_{n}^{m}(\theta) X_{n}^{m}\left(\theta^{\prime}\right)\right] f_{m}\left(\theta^{\prime}\right) \sin \theta^{\prime} d \theta^{\prime} \\
=\lambda f_{m}(\theta), \quad|m| \leq N, \quad 0 \leq \theta \leq \Theta .
\end{gathered}
$$

It should be noted that we can only obtain such eigenfunctions $f_{m}(\theta)$ for $|m| \leq N$ with nonzero eigenvalues $\lambda$. For those eigenfunctions with eigenvalues $\lambda=0$, they vanish in $\Gamma$ and have no effect in $0 \leq n \leq N$ [28]. After solving the above eigenvalue equation (28), the optimal associated spatial functions $f_{m}(\theta, \phi)$ with eigenvalues $\lambda_{m}>0$ are obtained by

$$
f_{m}(\theta, \phi)=f_{m}(\theta) e^{i m \phi} .
$$

Numerical Example: We use the same numerical parameters used in [34]. $N=18, \Theta=40^{\circ}$ and consider two cases of diffusion, $\xi=5 \times 10^{-4}$ and $\xi=5 \times 10^{-3}$. By applying the Gauss-Legendre quadrature method [44] to the homogeneous Fredholm equation, the distribution of the eigenvalues for $m=0,1, \ldots, 10$ with $\xi=5 \times 10^{-4}$ is shown in Fig. 2 . From this figure, we can observe that the eigenvalue decreases 


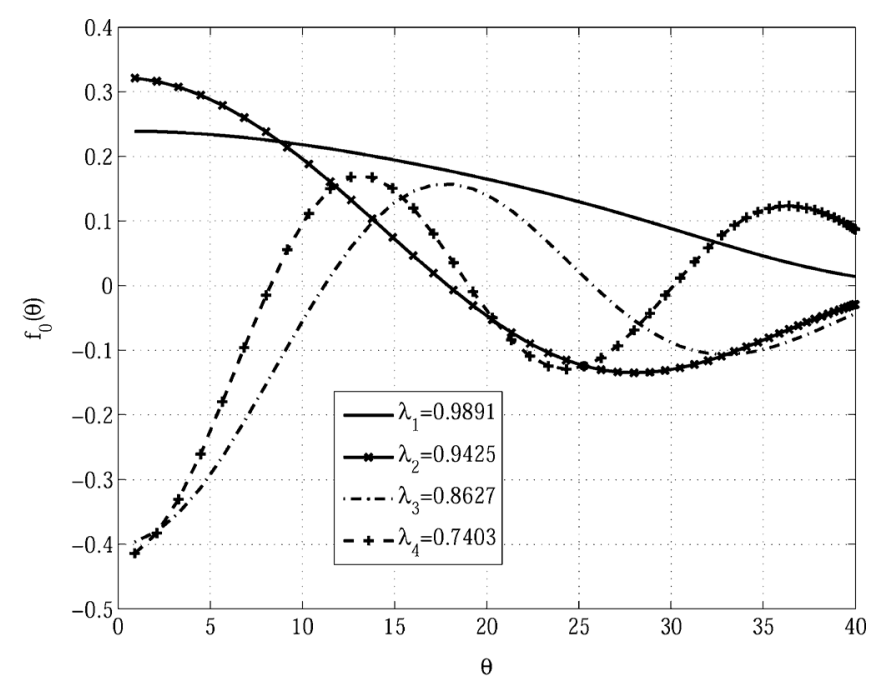

(a)

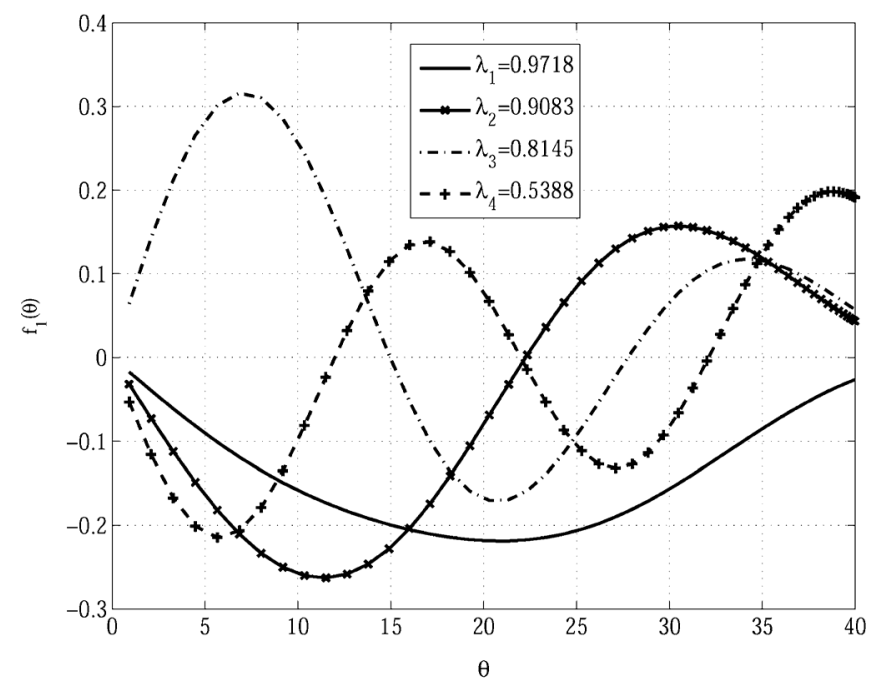

(b)

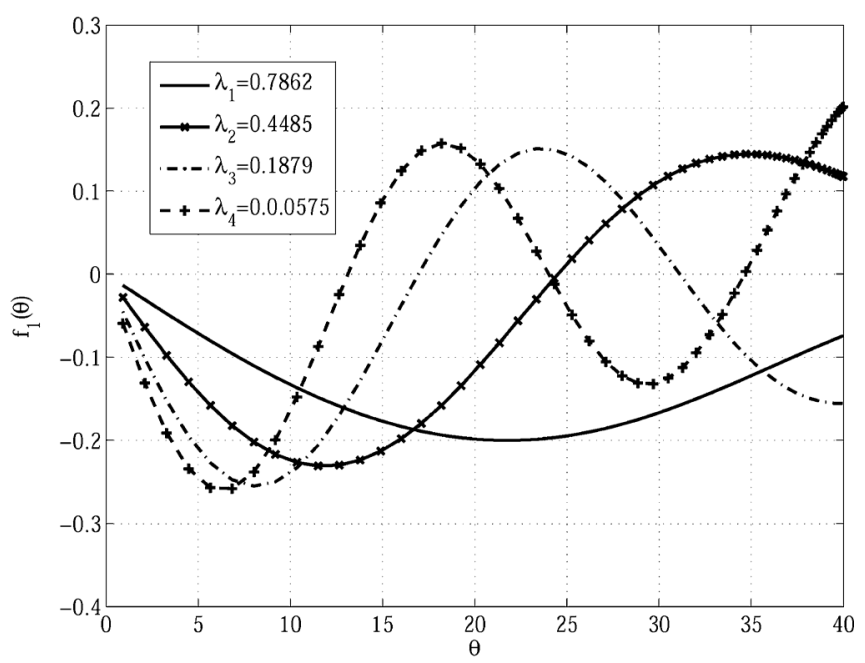

(c)

Fig. 3. Optimal eigenfunctions $f_{m}(\theta)$ obtained from (28) for $N=18, \Theta=$ $40^{\circ}$ : (a) $m=0$ with $\xi=5 \times 10^{-4}$, (b) $m=1$ with $\xi=5 \times 10^{-4}$, and $m=1$ with $\xi=5 \times 10^{-3} \cdot \lambda_{j}$ with rank $j=1,2,3,4$ are the corresponding eigenvalues of (28). (a) $m=0, \xi=5 \times 10^{-4}$, (b) $m=1, \xi=5 \times 10^{-4}$, (c) $m=1, \xi=5 \times 10^{-3}$.

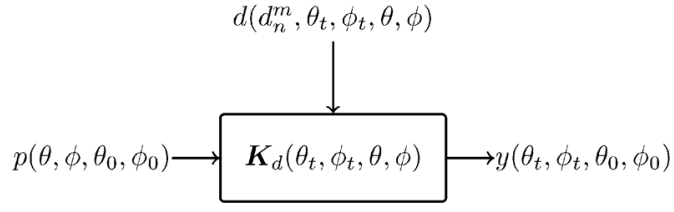

Fig. 4. A 3-D joint beamformer design. $p=p\left(k r, \theta, \phi, \theta_{0}, \phi_{0}\right)$ is the received wavefield at $(\theta, \phi)$ from a direction $\left(\theta_{0}, \phi_{0}\right), d\left(d_{n}^{m}, \theta_{t}, \phi_{t}, \theta, \phi\right)$ is the 3-D beamformer directing at $\left(\theta_{t}, \phi_{t}\right)$ which is controlled by the weighting sequence $\left\{d_{n}^{m}\right\}$ with $0 \leq n \leq N$ and $|m| \leq n$ for one specified integer $N$, and $\boldsymbol{K}_{d}\left(\theta_{t}, \phi_{t}, \theta, \phi\right)$ is the beamformer kernel.

as $m$ increases, and the maximum eigenvalue $\lambda_{0}=0.9891$ corresponds to the rank 1 of $m=0$. Therefore, the optimal spatial function is the one corresponding to $\lambda_{0}=0.9891$. Fig. 3 shows the optimal associated eigenfunctions $f_{m}(\theta)$ for $m=0,1$. These figures show that the eigenvalues for fixed $m$ decrease quickly as $\xi$ increases.

\section{A Joint 3-D Beamforming Scheme}

To further demonstrate the full power of our proposed framework, in this section we formulate a joint 3-D beamforming scheme for spherical-aperture microphone arrays. We demonstrate that a much more flexible optimal robust beamformer can be designed by taking different weighting function $w(\boldsymbol{x})$ and the operator $\mathcal{K}_{v}$ which represent different constraints through our framework.

Instead of maximizing the beamforming directivity only, as done in [45]-[47], our aim is to design a beamformer (or a filter) which also has good spatial resolution by achieving the maximum energy in the main lobe of the beamformer response, i.e., the spatial region $\Gamma \in \mathbb{S}^{2}$ around the direction $\left(\theta_{t}, \phi_{t}\right)$. In the following, for simplicity, we will assume $\Gamma$ is a cap with size $[0, \Theta]$. Here, we point out that our optimization problem is different from the current 3D beamforming optimization problems such as delay-and-sum (DAS) beam pattern [48] by adjusting the weights to compensate the delay, Dolph-Chebyshev beampattern [49] by exploiting the characteristics of the Chebyshev polynomials which minimizes the null-to-null main-lobe width for a given side-lobe level or the side-lobe level for a given null-to-null main-lobe width, and the minimum variance distortionless response (MVDR) beamformer [50].

The system diagram is shown in Fig. 4, where $p=$ $p\left(k r, \theta, \phi, \theta_{0}, \phi_{0}\right)$ is the received wave-field at $(r, \theta, \phi)$ from an incidental direction $\left(\theta_{0}, \phi_{0}\right)$ with $k$ being the wave number, $d=d\left(d_{n}^{m}, \theta_{t}, \phi_{t}, \theta, \phi\right)$ with $0 \leq n \leq N$ and $|m| \leq n$ is the 3-D beamformer directing at $\left(\theta_{t}, \phi_{t}\right)$ and controlled by the weights $\left\{d_{n}^{m}\right\}$ (or the Fourier coefficients of $d$ ). The beamformer kernel is given by

$$
\boldsymbol{K}_{d}\left(\theta_{t}, \phi_{t}, \theta, \phi\right)=\sum_{n=0}^{N} \sum_{m=-n}^{n} d_{n}^{m} Y_{n}^{m}\left(\theta_{t}, \phi_{t}\right) \overline{Y_{n}^{m}(\theta, \phi)}
$$

Using the model introduced in [46], we write the received wave field as

$$
p\left(k r, \theta, \phi, \theta_{0}, \phi_{0}\right)=\sum_{n=0}^{\infty} \sum_{m=-n}^{n} p_{n}^{m}\left(k r, \theta_{0}, \phi_{0}\right) Y_{n}^{m}(\theta, \phi)
$$


where

$$
\begin{aligned}
p_{n}^{m}\left(k r, \theta_{0}, \phi_{0}\right) & =b_{n}(k r) \overline{Y_{n}^{m}\left(\theta_{0}, \phi_{0}\right)} \\
b_{n}(k r) & =4 \pi i^{n}\left[j_{n}(k r)-\frac{j_{n}^{\prime}(k r)}{h_{n}^{\prime}(k r)} h_{n}(k r)\right],
\end{aligned}
$$

with $i=\sqrt{-1}, j_{n}(\cdot)$ and $j_{n}^{\prime}(\cdot)$ are the spherical Bessel functions and its derivative, $h_{n}(\cdot)$ and $h_{n}^{\prime}(\cdot)$ are the spherical Hankel function and its derivative, respectively. Then according to the kernel operation between the input $p\left(k r, \theta, \phi, \theta_{0}, \phi_{0}\right)$ and the 3-D beamformer kernel $\boldsymbol{K}_{d}\left(\theta_{t}, \phi_{t}, \theta, \phi\right)$, the output $y\left(\theta_{t}, \phi_{t}, \theta_{0}, \phi_{0}\right)$ is given by

$y\left(\theta_{t}, \phi_{t}, \theta_{0}, \phi_{0}\right)$

$$
=\sum_{n=0}^{N} \sum_{m=-n}^{n} b_{n}(k r) d_{n}^{m} \overline{Y_{n}^{m}\left(\theta_{0}, \phi_{0}\right)} Y_{n}^{m}\left(\theta_{t}, \phi_{t}\right) .
$$

For simplicity, we assume an omnidirectional 3-D beamformer, i.e., $d_{n}^{m}=d_{n}$ for all $|m| \leq n$. Therefore, the output $y(\boldsymbol{\Theta}) \triangleq$ $y\left(\theta_{t}, \phi_{t}, \theta_{0}, \phi_{0}\right)$ can be simplified to

$$
y(\boldsymbol{\Theta})=\sum_{n=0}^{N} b_{n}(k r) d_{n} \frac{2 n+1}{4 \pi} P_{n}(\cos \boldsymbol{\Theta})
$$

where $\Theta$ is the angle between $\left(\theta_{0}, \phi_{0}\right)$ and $\left(\theta_{t}, \phi_{t}\right)$. Assume $\left(\theta_{0}, \phi_{0}\right)$ is the north pole with $\theta_{0}=0$ and $\phi_{0}=0$. Then the output is totally controlled by the direction vector $\left(\theta_{t}, \phi_{t}\right)$. That is, $\boldsymbol{\Theta}$ can be written into a single point $\boldsymbol{x}=(\theta, \phi)$, and $y(\boldsymbol{x})=$ $\sum_{n=0}^{N} \sqrt{\frac{2 n+1}{4 \pi}} b_{n}(k r) d_{n} Y_{n}^{0}(\boldsymbol{x})$.

We now apply the variational framework (3) to determine the optimal choice of $d_{n}$ to achieve the maximum energy concentration in the main lobe, i.e., we maximize $I_{1}$ under the constraints $I_{2}$ and $I_{3}$, where

$$
\begin{aligned}
& I_{1}=\langle w y, y\rangle=\int_{\Gamma}|y(\boldsymbol{x})|^{2} d s(\boldsymbol{x}) \\
& I_{2}=\left\langle\mathcal{K}_{v} y, y\right\rangle=\sum_{n=0}^{N} \sum_{m=-n}^{n} v_{n}\left|y_{n}^{m}\right|^{2}=\sum_{n=0}^{N}\left|d_{n}\right|^{2} \\
& I_{3}=\langle y, y\rangle=\int_{\mathbb{S}^{2}}|y(\boldsymbol{x})|^{2} d s(\boldsymbol{x})=\sum_{n=0}^{N} \frac{2 n+1}{4 \pi}\left|b_{n} d_{n}\right|^{2}
\end{aligned}
$$

with $w(\boldsymbol{x})$ a box-car function representing the width of the main lobe of the 3D beamformer, $b_{n} \triangleq b_{n}(k r)$ and $v_{n}=\frac{4 \pi}{2 n+1} \frac{1}{b^{2}}$. Here, the constraint for a robust beamformer is implemented in $I_{2}$ by a sequence $\left\{v_{n}\right\}$ through the operator $\mathcal{K}_{v}$. When we take the coefficients of the operator $\mathcal{K}_{v}$ with $v_{n}=\frac{4 \pi}{2 n+1} \frac{1}{b_{n}^{2}}$, then $I_{2}$ represents the constraint of the weighting coefficients; when $\mathcal{K}_{v}$ is taken as a delta function, then $I_{2}$ is defined as the maximum directivity. The constraint $I_{3}$ means the total energy of the beamformer is limited.

$$
\mu_{1} \mathbf{W} \hat{\mathbf{Y}}+\mu_{2} \mathbf{K} \hat{\mathbf{Y}}+\mathbf{U} \hat{\mathbf{Y}}=0
$$

in the spectral domain, where $\hat{\mathbf{Y}}$ is the vector containing all the coefficients of $y(\boldsymbol{x})$. Let $A(\theta, \phi)=\sum_{n=0}^{m} d_{n} Y_{n}^{m}(\theta, \phi)$ be a directional filter which is controlled by the set of weighting sequence $\left\{d_{n}\right\}$. From this, we can observe ${ }^{1}$ that our beamformer is jointly controlled by both the input wavefield $p\left(\theta, \phi, \theta_{0}, \phi_{0}\right)$ through $b_{n}$ and the direction filter $A(\theta, \phi)$ through $d_{n}$.

\section{CONCLUSION}

In this paper, we developed a quadratic constrained variational framework on the 2-sphere. Two necessary conditions corresponding to the spatial domain and spectral domain of a stationary point to the quadratic variational problem were obtained, respectively. We demonstrated the applicability of the quadratic variational framework by the well known spatialspectral concentration work on the 2-sphere and the optimal function which maximized the filter energy and a joint 3 -D beamforming scheme.

\section{REFERENCES}

[1] D. Slepian and H. O. Pollak, "Prolate spheroidal wave functions, Fourier analysis and uncertainty-I," Bell Syst. Tech. J., vol. 40, pp. 43-63, Jan. 1961.

[2] H. J. Landau and H. O. Pollack, "Prolate spheroidal wave functions, Fourier analysis and uncertainty-II," Bell Syst. Tech. J., vol. 40, no. 1, pp. 65-84, Jan. 1961.

[3] H. J. Landau and H. O. Pollak, "Prolate spheroidal wave functions, Fourier analysis and uncertainty-III: The dimension of the space of essentially time- and band-limited signals," Bell Syst. Tech. J., vol. 41, pp. 1295-1336, Jul. 1962.

[4] L. E. Franks, Signal Theory. Englewood Cliffs, NJ: Prentice-Hall, 1969.

[5] G. Lachs, "Optimization of signal waveforms," IEEE Trans. Inf. Theory, vol. IT-9, pp. 95-97, Apr. 1963.

[6] A. Wyner and H. Landau, "Optimum waveform signal sets with amplitude and energy constraints," IEEE Trans. Inf. Theory, vol. IT-30, no. 4, pp. 615-622, Jul. 1984.

[7] A. Nuttall and F. Amoroso, "Minimum Gabor bandwidth of M orthogonal signals," IEEE Trans. Inf. Theory, vol. 14, no. 5, pp. 440-444, Jul. 1965.

[8] A. Nuttall, "Minimum rms bandwidth of $M$ time-limited signals with specified code or correlation matrix," IEEE Trans. Inf. Theory, vol. IT-14, no. 5, pp. 699-707, Sep. 1968

[9] A. Papoulis, "Minimum-bias windows for high-resolution spectral estimates," IEEE Trans. Inf. Theory, vol. IT-19, no. 1, pp. 9-12, Jan. 1973.

[10] L. Xu, C. Bi, X. Chen, and J. Chen, "Resolution enhancement of nearfield acoustic holography by interpolation using band-limited signal restoration method," Chinese Sci. Bull., vol. 53, no. 20, pp. 3142-3150, Oct. 2008.

[11] T. Bülow, "Spherical diffusion for surface smoothing and denoising," Comput. Inf. Sci. Dept., Univ. of Pennsylvania , Philadelphia, PA, Tech. Rep. (CIS), Dec. 2001.

[12] T. Bülow, "Spherical diffusion for 3D surface smoothing," IEEE Trans. Pattern Anal. Mach. Intell., vol. 26, no. 12, pp. 1650-1654, Dec. 2004.

[13] J. D. McEwen, M. P. Hobson, and A. N. Lasenby, "Optimal filters on the sphere," IEEE Trans. Signal Process., vol. 56, no. 8, pp. 3813-3823, Aug. 2008.

[14] J. L. Sanz, D. Herranz, and E. M. Gonzalez, "Optimal detection of sources on a homogeneous and isotropic background," Astrophys. J., vol. 552, pp. 484-492, May 2001.

[15] D. Herranz, J. L. Sanz, R. B. Barreiro, and E. M. Gonzalez, "Scaleadaptive filters for the detection/separation of compact sources," Astrophys. J., vol. 580, pp. 610-625, 2002.

[16] M. Tegmark, D. H. Hartmann, M. S. Briggs, and C. A. Meegan, "The angular power spectrum of BASTE 3B gamma-ray bursts," Astrophys. J., vol. 468, p. 214, Sep. 1996.

[17] E. Hivon, K. M. Gorski, C. B. Netterfield, B. P. Crill, S. Prunet, and F. Hansen, "Master of the CMB anisotropy power spectrum: A fast method for statistical analysis of large and complex CMB data sets," Astrophys. J., vol. 567, pp. 2-17, Mar. 2002.

[18] S. Mitra, A. S. Sengupta, and T. Souradeep, "CMB power spectrum estimation using noncircular beams," Phys. Rev. D., vol. 70, p. 103002, 2004 .

${ }^{1}$ Due to space limitations here we do not provide sufficient solutions, simulation results and performance comparison of our proposed beamformer with other beamforming strategies. We anticipate to present these results in a future publication. 
[19] G. Faÿ, F. Guilloux, M. Betoule, J. F. Cardoso, J. Delabrouille, and M. L. Jeune, "CMB power spectrum estimation using wavelets," Phys. Rev. D., vol. 78, no. 8, p. 20, 2008.

[20] A. S. Y. Poon, R. W. Broderson, and D. N. C. Tse, "Degrees of freedom in multiple-antenna channels: A signal space approach," IEEE Trans. Inf. Theory, vol. 51, no. 2, pp. 523-536, Feb. 2005.

[21] R. A. Kennedy and T. D. Abhayapala, "Spatial concentration of wavefields: Towards spatial information content in arbitrary multipath scattering," in Proc. 4th Austr. Commun. Theory Workshop (AusCTW), Melbourne, Australia, Feb. 2003, pp. 38-45.

[22] R. A. Kennedy, P. Sadeghi, T. D. Abhayapala, and H. M. Jones, "Intrinsic limits of dimensionality and richness in random multipath fields," IEEE Trans. Signal Process., vol. 55, no. 6, pp. 2542-2556, Jun. 2007.

[23] W. Freeden and M. Schreiner, "Non-orthogonal expansions on the sphere," Math. Methods Appl. Sci., vol. 18, pp. 83-120, 1995.

[24] F. J. Narcowich and J. D. Ward, "Nonstationary wavelets on the m-sphere for scattered data," Appl. Comput. Harmon. Anal., vol. 3, pp. 324-336, Oct. 1996.

[25] A. Albertella, F. Sansò, and N. Sneeuw, "Band-limited functions on a bounded spherical domain: The Slepian problem on the sphere," $J$. Geodesy, vol. 77, pp. 436-447, 1999.

[26] R. Pail, G. Plank, and W. D. Schuh, "Spatially restricted data distributions on the sphere: The method of orthonormalized functions and applications," J. Geodesy, vol. 75, pp. 44-56, 2001.

[27] L. Miranian, "Slepian functions on the sphere, generalized Gaussian quadrature rule," Inverse Problems, vol. 20, pp. 877-892, 2004.

[28] F. J. Simons, F. A. Dahlen, and M. A. Wieczorek, "Spatiospectral concentration on a sphere," SIAM Rev., vol. 48, pp. 504-536, 2006.

[29] N. L. Fernandez, "Optimally space-localized band-limited wavelets on $s^{q-1}, " J$. Comput. Appl. Math., vol. 199, pp. 68-79, Feb. 2007

[30] M. A. Wieczorek and F. J. Simons, "Minimum-variance multitaper spectral estimation on the sphere," J. Four. Anal. Appl., vol. 13, pp. 665-692, 2007.

[31] F. Guilloux, G. Faÿ, and J. F. Cardoso, "Practical wavelet design on the sphere," Appl. Comput. Harmon. Anal., vol. 26, pp. 143-160, Mar. 2009.

[32] L. Wei, R. A. Kennedy, and T. A. Lamahewa, "Signal concentration on unit sphere: An azimuthally moment weighting approach," in Proc. IEEE Int. Conf. Acoust., Speech, Signal Process. (IEEE ICASSP), Dallas, TX, Mar. 14-19, 2010, pp. 3698-3701

[33] L. Wei, R. A. Kennedy, and T. A. Lamahewa, "Signal concentration on unit sphere: A local $k$-th moment zenithal energy concentration measure," in Proc. 11th Aust. Commun. Theory Workshop (AusCTW), Canberra, Australia, Feb. 2010, pp. 97-101.

[34] L. Wei and R. A. Kennedy, "On spectral concentration of signals on the 2-sphere under a generalized moment weighting criterion," in Proc. Eur. Signal Process. Conf. (EUSIPCO), Aalborg, Denmark, Aug. 2010, p. 4.

[35] M. K. Chung, K. M. Dalton, L. Shen, A. C. Evans, and R. J. Davidson, "Weighted Fourier series representation and its application to quantifying the amount of gray matter," IEEE Trans. Med. Imag., vol. 26, no. 4, pp. 566-581, Apr. 2007.

[36] M. K. Chung, K. M. Dalton, and R. J. Davidson, "Tensor-based cortical surface morphometry via weighted spherical harmoic representation," IEEE Trans. Med.l Imag., vol. 27, no. 8, pp. 1143-1151, Aug. 2008.

[37] E. W. Hobson, The Thoery of Spherical and Ellipsoidal Harmonics. New York: Chelsea, 1931.

[38] R. A. Kennedy, T. A. Lamahewa, and L. Wei, "On azimuthally symmetric 2-sphere convolution," in Proc. 6th U.S./Aust. Joint Workshop on Defence Appl. Signal Process. (DASP), Sep. 26-30, 2009, p. 6

[39] K. Seon, "Smoothing of all-sky survey map with Fisher-von Mises function,” J. Korean Phys. Soc., vol. 48, no. 3, pp. 331-334, Mar. 2006.

[40] L. Debnath and P. Mikusinski, Introduction to Hilbert Spaces With Applications. San Diego, CA: Academic, 1990.

[41] A. R. Edmonds, Angular Momentum in Quantum Mechanics. Princeton, NJ: Princeton Univ. Press, 1996.

[42] F. A. Dahlen and F. J. Simons, "Spectral estimation on a sphere in geophysics and cosmology," Geophys. J. Int., vol. 174, pp. 774-807, Aug. 2008.

[43] J. R. Driscoll and D. M. H. , Jr., "Computing Fourier transforms and convolutions on the 2-sphere," Adv. Appl. Math., vol. 15, pp. 202-250, June 1994.

[44] W. H. Press, S. A. Teukolsky, W. T. Vetterling, and B. P. Flannery, Numerical Recipes in C: The Art of Scientific Computing. New York: Cambridge Univ. Press, 1988.
[45] B. Rafaely, "Plane-wave decomposition of the sound field on a sphere by spherical convolution," J. Acoust. Soc. Amer., vol. 116, no. 4, pp 2149-2157, Oct. 2004.

[46] B. Rafaely, "Analysis and design of spherical microphone arrays," IEEE Trans. Speech, Audio Process., vol. 13, no. 1, pp. 135-143, Jan. 2005.

[47] M. Agmon, B. Rafaely, and J. Tabrikian, "Maximum directivity beamformer for spherical-aperture microphones," in Proc. IEEE Workshop Appl. Signal Process. Audio Acoust., New Paltz, NY, Oct. 18-21, 2009 pp. $153-156$.

[48] B. Rafaely, "Phase-mode versus delay-and-sum spherical microphone array processing," IEEE Signal Process. Lett., vol. 12, no. 10, pp. 713-716, Oct. 2005.

[49] A. Koretz and B. Rafaely, "Dolph-Chebyshev beampattern design for spherical arrays," IEEE Trans. Signal Process., vol. 57, no. 6, pp. 2417-2420, Jun. 2009.

[50] S. F. Yan, H. H. Sun, U. P. Svensson, X. H. Ma, and J. M. Hovem, "Optimal modal beamforming for spherical microphone arrays," IEEE Trans. Audio Speech Lang. Process., vol. 19, no. 2, pp. 361-371, Feb. 2011.

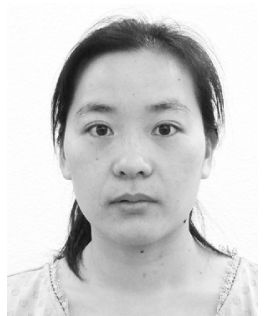

Liying Wei (S'09-M'11) received the B.E. degree from Xidian Unversity, China, in 2002, the M.E. degree from Beijing University of Posts and Telecommunications, Beijing, China, in 2006, and the $\mathrm{Ph} . \mathrm{D}$. degree from the Australian National University, Australia, in 2011

She worked as a Research Fellow in Applied Signal Processing Group, School of Engineering, the Australian National University from September 2010 to March 2011. She is currently a Postdoctora Fellow in the Department of Electronic Engineering, The Chinese University of Hong Kong. Her current research interest includes signal and image processing, statistical estimation and detection, wireless sensor networks, and wireless communications.

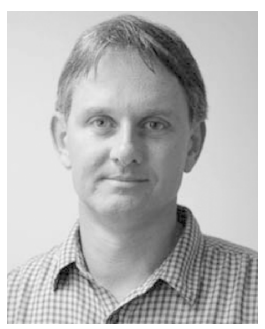

Rodney A. Kennedy (S'86-M'88-SM'01-F'05) received the B.E. degree from the University of New South Wales, Sydney, Australia, the M.E. degree from the University of Newcastle, and the Ph.D. degree from the Australian National University, Canberra.

He was with the Commonwealth Scientific and Industrial Research Organization (CSIRO) on the Australia Telescope Project for three years. He is currently a Professor and was the Head of the Department of Information Engineering, Research School of Information Sciences and Engineering, Australian National University. His research interests are in the fields of digital signal processing, spatial information systems, digital and wireless communications, and acoustical signal processing. More recently, his research has included more life science related to medical image processing and biological ion channel modeling.

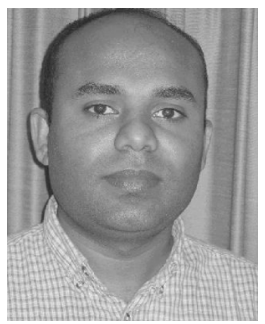

Tharaka A. Lamahewa (M'06) received the B.E. degree from the University of Adelaide, South Australia, in 2000 and the Ph.D. degree from the Australian National University, Canberra, in 2007.

He worked as a Software Design Engineer for two years at Motorola Electronics Pvt Ltd., Singapore. From 2006 to 2007, he worked as an Algorithm Design Engineer at Nanoradio AB, Melbourne, Australia. He is currently with the Applied Signal Processing Group, Research School of Information Sciences and Engineering, the Australian Nationa University. He is also a Wireless Researcher with National ICT Australia (NICTA). His research interests include information theory of time-varying fading channels, cooperative diversity, space-time coding and space-time-frequency channel modeling, body-area networks, underwater communications, and signal processing on the unit sphere. 